Without significant loss of generality we may take $\mu_{1}=1$. Determining $g(u), A(y), \psi(t)$ from the differential equations (14)(i), (ii) and the recurrence relation (12)(i) leads to known generating functions.

For $\alpha-\beta=0$ we have the ultraspherical polynomials with known generating functions of Appell type.

\title{
REFERENCE
}

1. G. Szegö, Orthogonal polynomials, Amer. Math. Soc. Colloquium Publications, vol. $23,1939$.

\section{APPROXIMATION BY FAMILIES OF FUNCTIONS ${ }^{1}$}

\section{LEONARD TORNHEIM}

Let $F$ be a set of real functions on the interval $a \leqq x \leqq b$ and let $g(x)$ be a real and continuous function on the same interval. The problem of finding a function in $F$ which approximates $g$ best according to some criterion has been extensively studied, especially in the two cases when $F$ is the set of all real polynomials of degree less than $n$ or a fixed set of trigonometric functions $[1 ; 2 ; 4$, pp. 40-41]. We investigate the problem for $n$-parameter families of functions [5] with reference to some of the usual approximation criteria.

An $n$-parameter family $F$ of functions on the interval $a \leqq x \leqq b$ is a set of real continuous functions $f$ such that for every set of points $\left(x_{i}, y_{i}\right)(i=1, \cdots, n)$ with $a \leqq x_{1}<\cdots<x_{n} \leqq b$ there exists exactly one $f$ in $F$ with $f\left(x_{i}\right)=y_{i}(i=1, \cdots, n)$.

An $n$-parameter family is linear if it is a real vector space, i.e., if there exist $n$ functions $f_{1}, \cdots, f_{n}$ such that every $f$ may be expressed as a linear combination $f=a_{1} f_{1}+\cdots+a_{n} f_{n}$, where $a_{1}, \cdots, a_{n}$ are real.

For $k \geqq 1$ the modulus of approximation $M^{(k)}$ of $g$ by $f$ is defined as

$$
\begin{array}{ll}
M^{(k)}(f) & =\int_{a}^{b}|f-g|^{k} d x \quad(k<\infty), \\
M^{(\infty)}(f) & =\max |f-g| .
\end{array}
$$

Further a function $f_{0}$ in $F$ will be called a best $k$-approximant to $g$ if

Presented to the Society, November 27, 1954; received by the editors August 1, 1955.

${ }^{1}$ Supported by a grant from the National Science Foundation. 


$$
M^{(k)}\left(f_{0}\right)=\underset{f \in F}{\text { g.l.b. }} M^{(k)}(f) .
$$

The existence and uniqueness of a best $\infty$-approximant was demonstrated in [5, Theorems 7 and 9].

Theorem 1. $A$ best k-approximant always exists.

Let $f_{1}, f_{2}, \cdots$ be a sequence of functions (in the $n$-parameter family $F$ ) such that $\lim _{i \rightarrow \infty} M^{(k)}\left(f_{i}\right)=m$, where $m=$ g.l.b. $M^{(k)}(f)$. Let $d=(b-a) / 3(n+1)$, and let $x_{j}=a+3 j d(j=1,2, \cdots, n)$. On the interval $x_{j}-d \leqq x \leqq x_{j}+d$, let the minimum of $\left|f_{i}-g\right|$ occur at $x_{i j}$ and set $y_{i j}=f_{i}\left(x_{i j}\right)$. Since the sequence $\left\{M^{(k)}\left(f_{i}\right)\right\}(i=1,2, \cdots)$ is bounded the set of values $y_{i j}$ is bounded. Hence for each $j$, the points $\left(x_{i j}, y_{i j}\right)$ have a limit point $\left(x_{j}, y_{j}\right)$. Hence there is a subsequence $\left\{f_{i^{\prime}}\right\}$ of the sequence $\left\{f_{i}\right\}$ such that $\lim _{i^{\prime} \rightarrow \infty}\left(x_{i^{\prime} j}, y_{i^{\prime} j}\right)=\left(x_{j}, y_{j}\right)$ $(j=1, \cdots, n)$. Consequently, if $f$ is the function in $F$ through $\left(x_{1}, y_{1}\right), \cdots,\left(x_{n}, y_{n}\right), \lim _{i^{\prime} \rightarrow \infty} f_{i^{\prime}}=f$ uniformly [5, Theorem 5, p. 460]; thus $M^{(k)}\left(f_{i^{\prime}}\right) \rightarrow M^{(k)}(f)$, and so $f$ is a best $k$-approximant.

TheOREM 2. $A$ best $k$-approximant is unique if $F$ is linear and $k>1$.

When $k$ is an even integer, the proof of Pólya [3] for the special case of polynomials is also effective here.

In general we use the strict convexity of $y=|x|^{k}$. If $f_{1} \neq f_{2}$ and $f=\left(f_{1}+f_{2}\right) / 2$ then $|f-g|^{k} \leqq\left(\left|f_{1}-g\right|^{k}+\left|f_{2}-g\right|^{k}\right) / 2$, with inequality at some point; thus, since the functions are continuous $M^{(k)}(f)$ $<\left[M^{(k)}\left(f_{1}\right)+M^{(k)}\left(f_{2}\right)\right] / 2$. Hence the best approximant is unique. Were $k=1$ all that we could say on the basis of this proof is that $\left(f_{1}-g\right)\left(f_{2}-g\right) \geqq 0$ if $f_{1}$ and $f_{2}$ are best 1-approximants.

THEOREM 3. If $n=1, a$ best $k$-approximant need not be unique for $k \neq \infty$. Also it need not cross $g$.

We give an example for $k=1$. Let $a=0, b=1$. Let $g(x)=0$. We designate by $f_{r}$ that $f$ in $F$ for which $f(0)=r$, i.e., by its $y$-intercept. Let $f_{r}$ for $r \leqq-1$ be the line segment of slope 1 ; for $-1 \leqq r \leqq-1 / 2$ the pair of line segments, one joining $(0, r)$ to $(s, s+r)$ having slope 1 and the other $(s, s+r)$ to $(1,3-2 s+r)$ having slope 3 , where $s=3+2 r$ $-\left[3\left(r^{2}+3 r+2\right)\right]^{1 / 2}$; and for $-1 / 2 \leqq r, f_{r}=f_{-1 / 2}+r+1 / 2$. Then all $f_{r}$ $(-1 \leqq r \leqq-1 / 2)$ are best 1 -approximants to $g$ and $f_{-1}$ does not cross $g$. Only a slight change in the definition of $s$ is needed to take care of other values of $k$.

THEOREM 4. Let $f_{k}$ be a sequence of best $k$-approximants where $k \rightarrow \infty$. Then $\lim _{k \rightarrow \infty} f_{k}=f_{\infty}$. 
The proof of Pólya [3] for the case of polynomials actually takes care of the general case after we have proved that there is a subsequence of the $f_{k}$ which approaches a limit $f^{*}$ uniformly. This can be done as in the proof of Theorem 1 after we have shown that $M^{(k)}\left(f_{k}\right)$ is bounded. But this fact was also proved by Pólya as follows:

$$
\begin{gathered}
M^{(k)}\left(f_{k}\right) \leqq M^{(k)}\left(f_{\infty}\right) \leqq\left[M^{(\infty)}\left(f_{\infty}\right)\right]^{k}(b-a) . \\
\text { REFERENCES }
\end{gathered}
$$

\section{REFERENCES}

1. S. Bernstein, Lecons sur les propriêtes extrémales et la meilleure approximation des fonctions analytiques d'une variable reelle, 1926.

2. C. J. de la Vallee Poussin, Leçons sur l'approximations des fonctions d'une variable réelle, 1919.

3. G. Polya, Sur un algorithme toujours convergent pour obtenir les polynomes de Tchebychef pour une fonction continue quelconque, C. R. Acad. Sci. Paris vol. 157 (1913) pp. 840-843.

4. G. Szegö, Orthogonal polynomials, 1939.

5. L. Tornheim, On n-parameter families of functions and associated convex functions, Trans. Amer. Math. Soc. vol. 69 (1950) pp. 457-467.

University of California, Berkeley 